\title{
IMPLANTAÇÃO DE DISPOSITIVO DE CORREÇÃO DE TELESCOPICIDADE EM BOBINAS A QUENTE NO LTQ DA ARCELORMITTAL TUBARÃO'
}

\author{
Sergio de Oliveira Lima Júnior ${ }^{2}$ \\ James William Laranja Ribeiro ${ }^{3}$ \\ João Augusto Guilherme de Barros ${ }^{4}$ \\ Ricardo de Araújo França ${ }^{5}$ \\ Michel de Fraipont ${ }^{6}$ \\ Luiz Roberto Zorzanelli ${ }^{7}$
}

\section{Resumo}

A telescopicidade é um defeito de forma de bobina caracterizada pelo deslocamento transversal ao sentido de laminação/bobinamento, de uma ou mais espiras, que pode ser causada por uma série de variáveis do processo de produção de tiras a quente. Cada cliente - seja ele interno ou externo - tem uma tolerância de aceite para a medida desta telescopicidade em seu pedido. Caso o valor ultrapasse esse limite, a bobina necessita ser reprocessada em linhas posteriores ao LTQ, como por exemplo, um laminador de acabamento ou linha de tesouras, antes de ser despachada para o cliente final. Com o objetivo de reduzir o retrabalho de bobinas - processo este que somente agrega custo ao produto - foi desenvolvido e implantado no LTQ da ArcelorMittal Tubarão um dispositivo de correção de telescopicidade. Este trabalho tem por finalidade apresentar os resultados atingidos após a implantação desta inovação tecnológica.

Palavras-chave: Telescopicidade; Laminação; Forma; Bobinas a quente.

\section{TELESCOPICITY CORRECTOR: TECHNOLOGICAL INOVATION AT ARCELORMITTAL TUBARÃO HOT STRIP MILL}

\begin{abstract}
Telescopicity is a coil defect characterized by transversal movement of wraps considering the direction of rolling or coiling. It can be caused by several process parameters during hot strip production. Each customer has his own tolerance concerning telescopicity maximum amount into his order. In case of the result of telescopicity exceed the acceptable value the coil has to be reworked at finishing lines before being dispatched to the client. It means to add unnecessary costs to the product. This paper presents the result achieved after the implementation of telescopicity corrector device at ArcelorMittal Tubarão hot strip mill, a technological innovation.
\end{abstract}

Key words: Telescopicity; Hot rolling; Hot coils; Coil shape.

\section{INTRODUÇÃO}

$\mathrm{Na}$ laminação de tiras a quente, a bobina - seu produto final - pode apresentar algum defeito de forma, à saber: empeno lateral, ondulações (má planicidade), ponta/cauda irregular, bobinamento frouxo, ovalização ou telescopicidade. Este último é o defeito de forma mais comum em bobinas a quente e é caracterizado pelo deslocamento transversal ao sentido de laminação/bobinamento, de uma ou mais espiras. A Figura I apresenta uma bobina com o defeito de forma que será abordado neste trabalho.

A telescopicidade, ou simplesmente TE, pode ocorrer em posições diferentes e, por isso, ela é classificada por tipo na ArcelorMittal Tubarão:

- tipo "A": primeiras espiras ou início de bobinamento (Figura I);

- tipo "B": últimas espiras ou fim de bobinamento; e

\footnotetext{
' Contribuição Técnica ao $43^{\circ}$ Seminário de Laminação, Processos e Produtos Laminados e Revestidos, 17 a 20 de Outubro de 2006, Curitiba, Paraná, Brasil

${ }^{2}$ Gerente de Área de Laminação de Tiras a Quente, ArcelorMittal Tubarão, Serra-ES

${ }^{3}$ Engenheiro Especialista em Engenharia Elétrica e Eletrônica, ArcelorMittal Tubarão, Serra - ES

${ }^{4}$ Engenheiro Especialista em Manutenção e Controle de Processo de LTQ, ArcelorMittal Tubarão, Serra - ES

${ }^{5}$ Engenheiro Especialista em Engenharia Mecânica, ArcelorMittal Tubarão, Serra - ES

${ }^{6}$ Engenheiro Especialista em Manutenção Mecânica de LTQ, ArcelorMittal Tubarão, Serra - ES

${ }^{7}$ Engenheiro Especialista em Automação e Controle de Processo, ArcelorMittal Tubarão, Serra - ES
} 
- tipo "Zig Zag": ao longo do "corpo" da bobina.

Dentre os tipos citados, a telescopicidade tipo "A" ocorre com maior freqüência, podendo variar de acordo com as condições de processo e dimensões do material que está sendo produzido. Portanto, com foco na redução desta ocorrência, e, conseqüentemente, diminuição do índice de bobinas com necessidade de retrabalho é que foi criado, desenvolvido e implantado o dispositivo de correção de telescopicidade no LTQ da ArcelorMittal Tubarão.

\section{FATORES INFLUENTES}

Durante a produção das tiras a quente uma série de variáveis de processo podem influenciar a

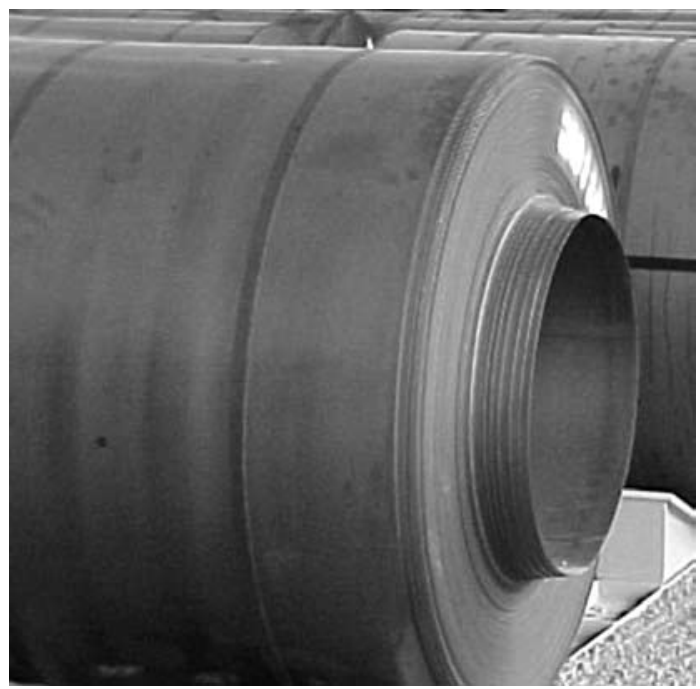

Figura I. Bobina a quente apresentando telescopicidade. ocorrência da telescopicidade interna ou tipo "A". A Figura 2 mostra quais são os fatores que contribuem para geração deste desvio.

\section{DESENVOLVIMENTO APÓS START-UP DO LTQ}

Após o início de operação do LTQ da ArcelorMittal Tubarão, ocorrido em $31 / 08 / 2002$, vários ajustes de parâmetros de processo foram implementados, com base no diagrama da Figura 2, visando a redução dos valores de telescopicidade em bobinas. Além disso, padrões internos de aceite e julgamento deste defeito de forma também sofreram alterações e, atualmente, variam de $30 \mathrm{~mm}$ a $50 \mathrm{~mm}$ (máximo) em função do cliente, modal de transporte, espessura e tipo de telescopicidade (A, B ou Zig-Zag).

O LTQ da ArcelorMittal Tubarão tem a característica de não ter associado ao mesmo, à sua frente, decapagem e laminação a frio. Com isso, é visado o mínimo valor de telescopicidade que garanta que a bobina chegue ao cliente com a menor avaria possível em função das subseqüentes movimentações que a bobina sofre até chegar ao seu destino final.

Contudo, por mais que se invista em ajustes operacionais, há um limite para a redução da ocorrência de telescopicidade interna, ou tipo "A".

Como existem muitos fatores que influenciam a ocorrência desta telescopicidade e seu controle é difícil, principalmente quando se tem um mix de produto variado - como é o caso do LTQ da ArcelorMittal Tubarão, onde o percentual de tiras menores ou iguais a $2 \mathrm{~mm}$ de espessura chega a quase $30 \%$. No primeiro semestre de 2004 foram avaliadas duas alternativas para reduzir o índice de bobinas desviadas para retrabalho pelo defeito telescopicidade: a primeira seria contratar uma assistência técnica externa onde fosse realizada uma transferência de know-how; a segunda seria de desenvolver internamente um dispositivo que fizesse a correção deste defeito, liberando a bobina para o seu fluxo de produção normal. Esta inovação teria o caráter de criação de know-how. Optou-se, então, pela segunda alternativa, sendo desenvolvido o corretor de telescopicidade em laminadores de tiras a quente.

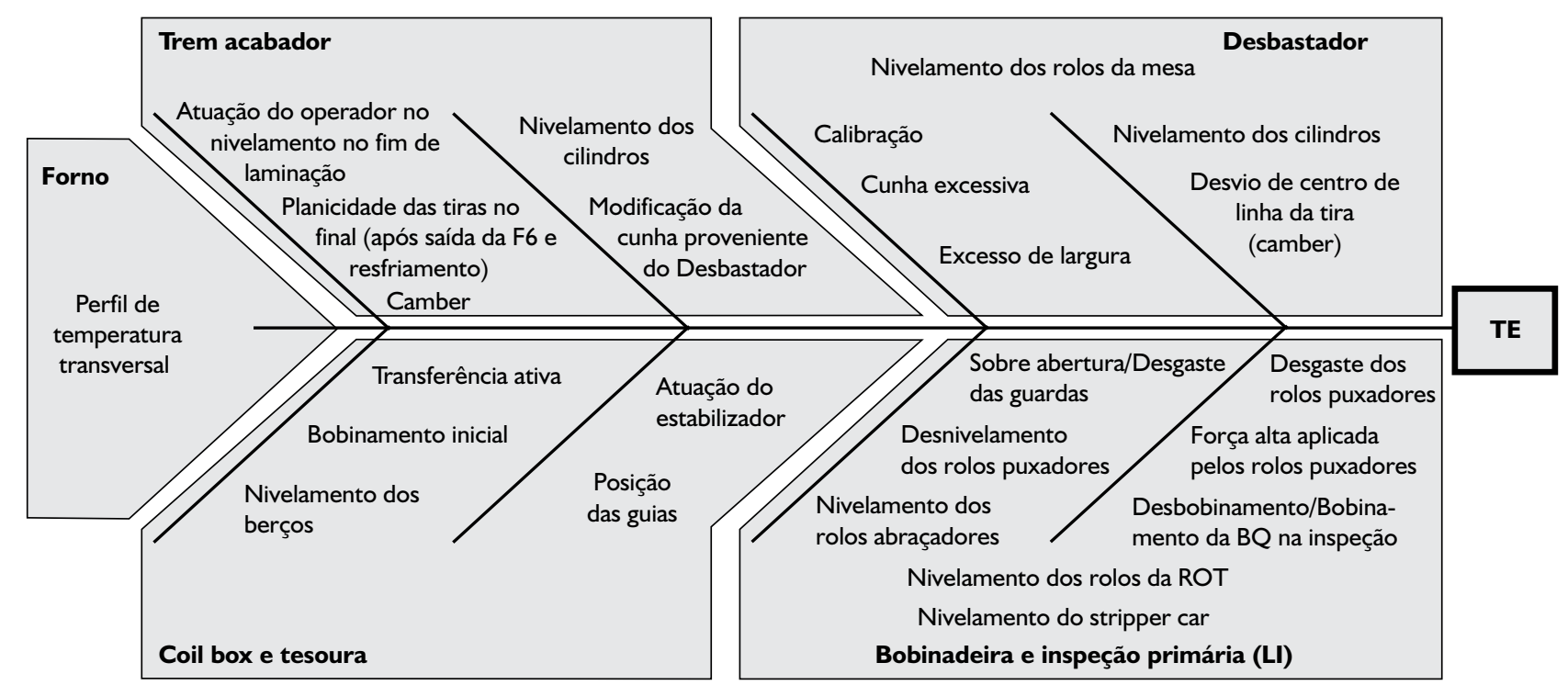

Figura 2. Diagrama de causa e efeito para ocorrência de telescopicidade. 


\section{O CORRETOR DE TELESCOPICIDADE}

\section{I Discussão Inicial e Concepção do Projeto}

Uma vez tomada a decisão do que deveria ser feito, uma equipe envolvendo diversas áreas (operação, engenharia elétrica $e$ eletrônica, engenharia de automação, engenharia mecânica e civil, manutenção elétrica e controle de processo e manutenção mecânica) foi formada para viabilizar a implantação do projeto.

Como itens básicos para este desenvolvimento a equipe procurou discutir sobre:

- a existência de equipamentos similares no mundo, onde foram encontrados alguns, contudo para correção de telescopicidade em bobinas transportadas com eixo na vertical e não horizontal como é o caso da ArcelorMittal Tubarão;

- a posição de instalação do dispositivo sob os aspectos de tempo de correção total do defeito, investimento necessário e condições de avaliação da telescopicidade final pelos inspetores de qualidade;

- o tamanho do dispositivo;

- a integração deste equipamento aos sistemas de automação e controle existentes no LTQ; e

- a análise e disponibilização dos resultados através do nível 3.

\subsection{Detalhamento do Projeto}

Discutidos e definidos todos estes pontos, coube as áreas envolvidas o desenvolvimento do projeto com o seguinte escopo:

- operação: expectativa de resultado, especificação técnica, descrição funcional e fluxo de processo;

- mecânica: dimensionamento do equipamento (função das forças atuantes), projeto detalhado (estrutural, mecânico e hidráulico);

- civil: projeto detalhado (bases dos equipamentos);

- elétrica: projeto e instalação de instrumentos, cabos e sensores;

- controle: descrição funcional e especificação dos equipamentos, testes de plataforma junto ao fornecedor, testes de campo e comissionamento; e
- automação: especificação técnica (interface com sistemas de nível I, nível 2 e nível 3 e interface com operador).

\subsection{Implantação e Comissionamento}

As etapas desenvolvidas até a utilização plena do equipamento são mostradas na Figura 3.

\subsection{Princípio de Funcionamento}

O corretor de telescopicidade utiliza cilindros hidráulicos para realizar a correção do defeito, ou seja, o enquadramento do seu valor dentro dos requisitos do cliente, evitando assim danos de manuseio, descarte de material e, principalmente, retrabalho. A Figura 4 mostra o dispositivo em processo de correção.

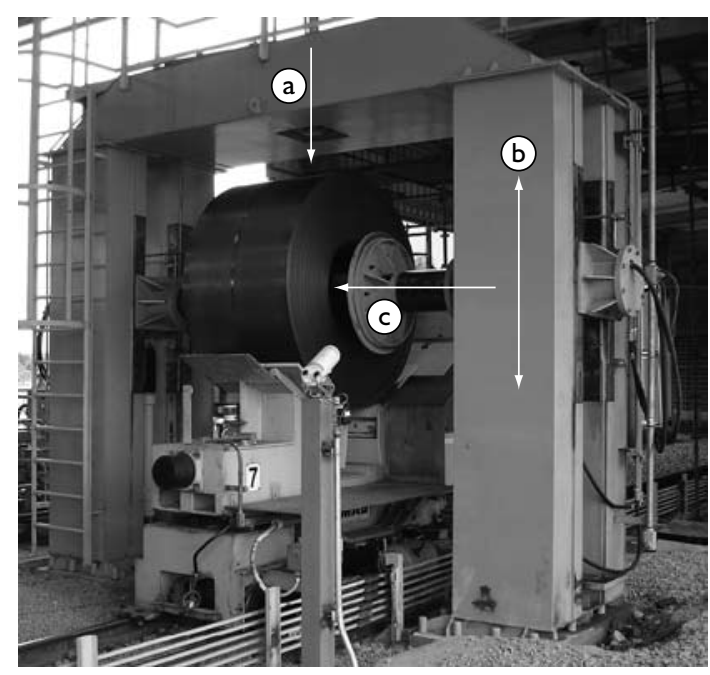

Figura 4. a) Detecção do diâmetro da bobina; b) Ajuste vertical dos discos de correção; e c) Movimento horizontal dos discos para correção da telescopicidade.

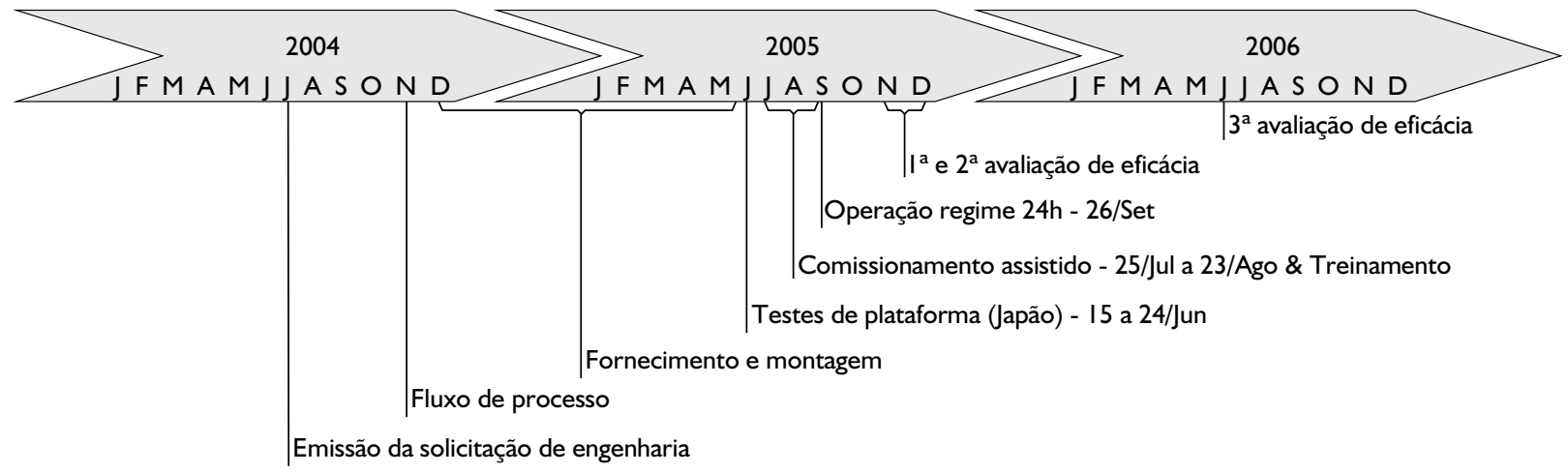

Figura 3. Cronologia do desenvolvimento do projeto de implantação do corretor. 
Movimento horizontal dos discos para correção da telescopicidade.

A condição em que a telescopicidade é observada na bobina determina o nível de dificuldade ou facilidade com a qual a mesma será corrigida. Como exemplo, quanto maior a espessura da tira e o número de espiras deslocadas e ainda quanto mais justo estiver o bobinamento das primeiras voltas, mais difícil o enquadramento do valor de telescopicidade nos limites definidos. A Figura 5 mostra uma seqüência de correção de telescopicidade em uma bobina, onde o valor final obtido atendeu a especificação do cliente.

A comunicação entre os sistemas de nível I, 2 e 3 é feita de forma automática e integrada, permitindo o monitoramento completo dos equipamentos e parâmetros através do PLC e supervisório de operação no púlpito de controle das bobinadeiras, que proporcionam o diagnóstico de todo sistema elétrico e de controle. A Figura 6 mostra como é feita esta comunicação entre os diversos sistemas.

Apesar do equipamento ficar localizado a $500 \mathrm{~m}$ de distância do operador responsável por seu controle, o uso de câmeras digitais o ajuda a monitorar o processo de correção.

\section{RESULTADOS OBTIDOS}

Apesar do ineditismo do projeto, em sua concepção, foi definida uma meta de enquadramento do valor de TE em, no mínimo, $90 \%$ das bobinas que sofrem correção, cuja rota é o cliente externo diretamente. A eficácia do equipamento é calculada pela relação entre $\circ$ " $n$ ' de BQ's com TE final < máx" e o " $n$ ' de BQ's indicadas para correção". Esta avaliação leva em consideração somente bobinas com ciclo completo de correção e que possuem rota direta para o cliente externo.

Em sua primeira avaliação, a eficácia do corretor de telescopicidade atingiu 92\%. $\mathrm{Na}$ segunda, $89 \%$ e na terceira $99 \%$.

De acordo com a Figura 7, a ArcelorMittal Tubarão, após a implantação do corretor de telescopicidade reduziu o retrabalho de $29.000 \mathrm{t} / \mathrm{mês}$, em média, para $4.000 \mathrm{t} / \mathrm{mês}$, mesmo com o aumento do volume de produção em $40 \%$ entre 2004 e 2006.

A redução de retrabalho em $25.000 \mathrm{t} / \mathrm{mês}$ proporcionou uma economia anual de US\$ $1.000 .000,00$ para a ArcelorMittal Tubarão.
A utilização do corretor de TE não gera qualquer desvio quanto à qualidade da superfície das espiras corrigidas.

Como este equipamento é uma inovação tecnológica, o mesmo encontra-se com patente depositada no INPI - Instituto Nacional da Propriedade Industrial - desde Setembro de 2005.
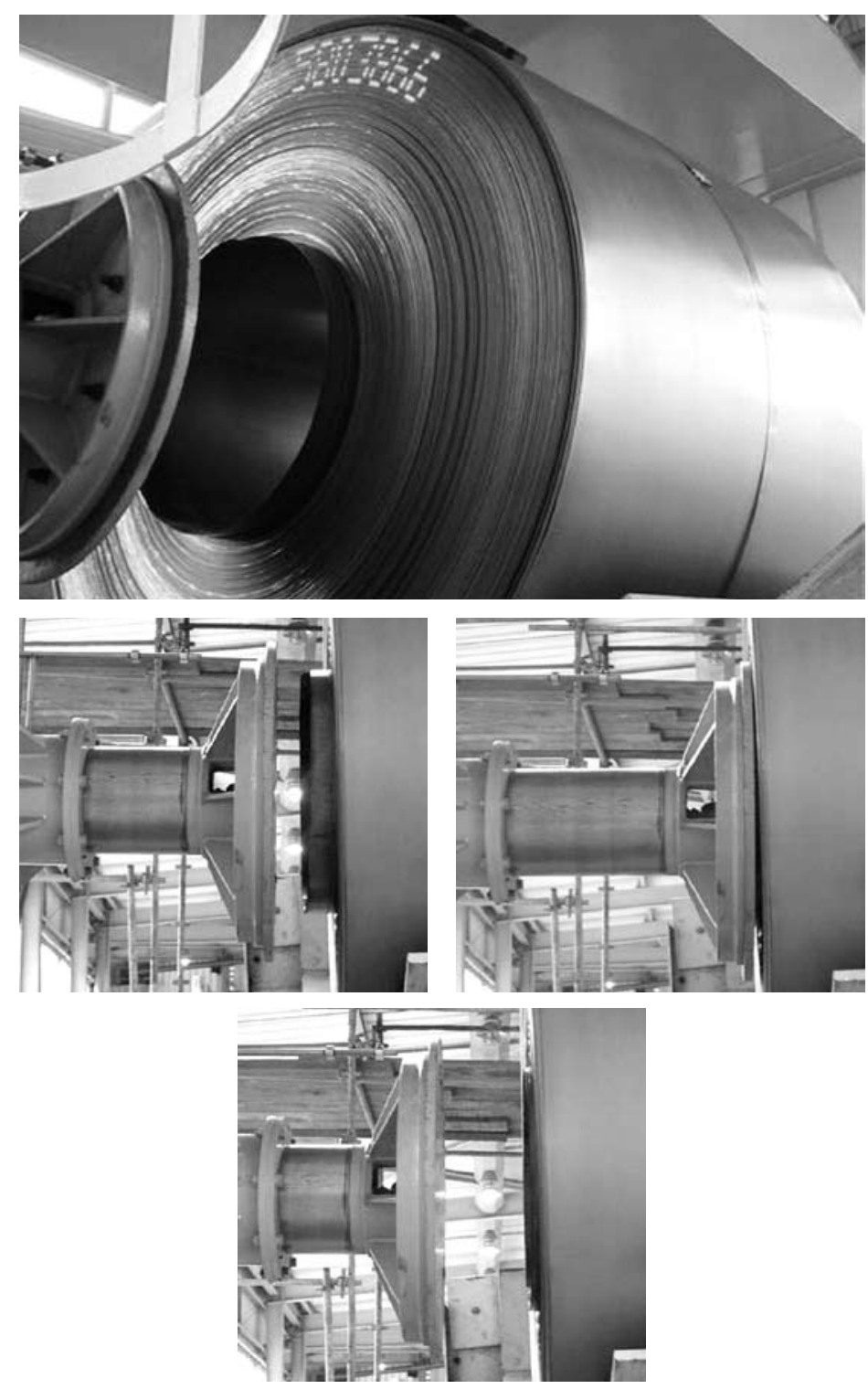

Figura 5. Bobina de uma tira com 2 × $1200 \mathrm{~mm}$ em processo de correção de TE.

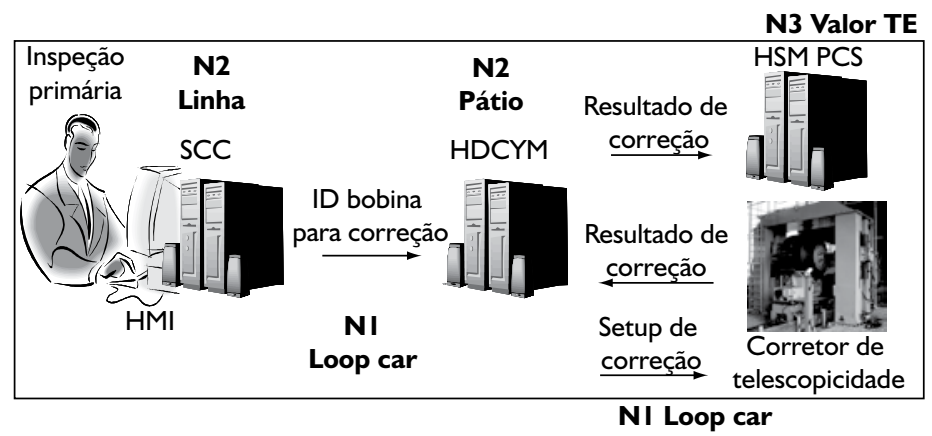

Figura 6. Comunicação entre os sistemas.

Tecnologia em Metalurgia e Materiais, São Paulo, v.3, n.3, p. 7-II, jan.-mar. 2007 


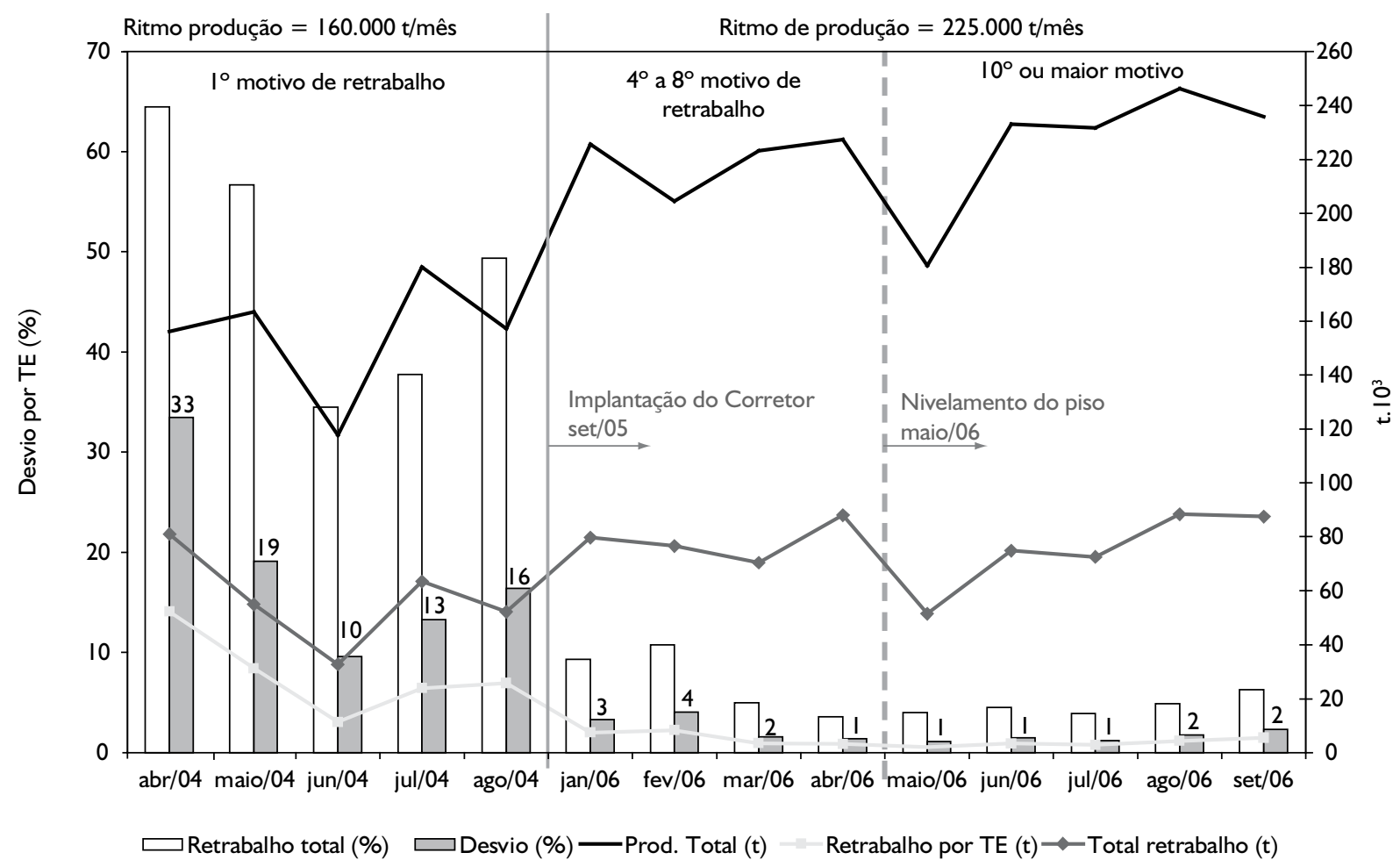

Figura 7. Redução do retrabalho por TE após a implantação do corretor.

\section{CONCLUSÕES}

- Apesar do ineditismo do projeto a meta estabelecida foi alcançada: enquadramento do valor de TE em, no mínimo, $90 \%$ das bobinas que sofreram correção, cuja rota é o cliente externo diretamente;

- não há prejuízo à qualidade superficial da tira;

- grande redução de custo para a empresa: US\$ I Mi/ano;

- manutenção do "lead time" de entrega do produto ao cliente final;

- sistema com monitoramento dos equipamentos e parâmetros através do PLC e supervisório de operação no púlpito de controle das bobinadeiras, que proporcionam o diagnóstico de todo sistema elétrico e de controle;

- melhoria do IBB (Índice de Bobina Boa); e

- eliminação do procedimento de correção de TE utilizando ponte rolante.

\section{Agradecimentos}

- Aos gerentes que nos propiciaram mais um desafio;

- aos projetistas que conceberam o dispositivo;

- a TMEIC que auxiliou na elaboração da lógica;

- as manutenções/engenharias mecânica e elétrica e automação que suportaram com informações precisas a engenharia básica e detalhada do projeto até a sua completa implementação;

- aos operadores que participaram ativamente da fase de implantação, fazendo com que o equipamento tenha e seja um valor agregado ao processo; e

- aos inspetores que indicam as bobinas para correção e realizam a inspeção final no pátio BQ-0 (atualmente).

Recebido em: 18/01/07

Aceito em: 17/04/07

Proveniente de: SEMINÁRIO DE LAMINAÇÃO - PROCESSOS E PRODUTOS LAMINADOS E REVESTIDOS, 43., 2006, Curitiba. São Paulo: ABM, 2006. 